\title{
Qui veut garder ses millions? Redistribution des aides dans la nouvelle PAC
}

\section{Pauline Lécole et Sophie Thoyer}

\section{(2) OpenEdition}

1 Journals

\section{Édition électronique}

URL : http://journals.openedition.org/economierurale/4701

DOI : 10.4000/economierurale.4701

ISSN : 2105-2581

\section{Éditeur}

Société Française d'Économie Rurale (SFER)

\section{Édition imprimée}

Date de publication : 30 septembre 2015

Pagination : 59-79

ISSN : 0013-0559

\section{Référence électronique}

Pauline Lécole et Sophie Thoyer, « Qui veut garder ses millions ? Redistribution des aides dans la nouvelle PAC », Économie rurale [En ligne], 348 | juillet-août 2015, mis en ligne le 01 janvier 2017, consulté le 10 décembre 2020. URL : http://journals.openedition.org/economierurale/4701 ; DOI https://doi.org/10.4000/economierurale.4701

(c) Tous droits réservés 


\title{
FAITS ET CHIFFRES
}

\section{Qui veut garder ses millions ? Redistribution des aides dans la nouvelle PAC}

\author{
Pauline LÉCOLE, Sophie THOYER • Montpellier Supagro, UMR Lameta \\ lecole@supagro.fr ; thoyer@supagro.fr
}

La Commission européenne dans sa proposition de réforme de la Politique agricole commune a déclaré vouloir introduire plus d'équité dans la distribution des aides agricoles du premier pilier entre États-membres et au sein des États-membres. Cet article analyse la dispersion des soutiens directs dans la situation avant réforme. Une décomposition de l'indice de Theil démontre qu'elle est plus liée à la distribution au sein des États-membres qu'entre les États-membres. Les auteurs la caractérisent dans les deux cas et présentent les notions d'équité qui sous-tendent I'accord politique obtenu entre le Parlement européen, le Conseil et la Commission européenne. Les mesures négociées dans cet accord - la convergence des montants des droits à paiements de base, la dégressivité et le paiement redistributif - sont présentées et illustrées dans le cas français. Enfin, l'analyse du processus dit de " convergence externe ", c'est-à-dire de transferts en faveur des États-membres ayant des montants d'aides directes par hectare plus faibles que la moyenne, conclut sur des changements marginaux en termes de montants et de bénéficiaires concernés.

MOTS-CLÉS : aides directes, politique agricole commune, iniquité de distribution, indice de Gini, indice de Theil

$\mathbf{L}$ 'accord politique sur la réforme de la Politique agricole commune (PAC) pour la programmation 2014-2020 a été trouvé en juin 2013 après deux années d'intenses négociations en particulier sur les modalités de redistribution des aides du premier pilier. L'une des grandes ambitions affichées de la nouvelle PAC est en effet d'instituer une logique de répartition des soutiens directs différente de celle adoptée lors de la réforme à mi-parcours de 2003.

Dès sa communication de novembre 2010, la Commission européenne (CE) a souligné « les nécessaires adaptations du système de paiements directs consistant en la redistribution, le réaménagement et un meilleur ciblage du soutien, destinées à accroître la valeur et la qualité des

1. Ce travail a bénéficié d'une aide de l'État gérée par l'Agence Nationale de la Recherche au titre du programme «Investissements d'avenir » portant la référence ANR-10-LABX-0001-01 avec Agropolis Fondation (projet CAPeye : https:// www.supagro.fr/capeye/). dépenses » $(\mathrm{CE}, 2010)^{2}$. Elle annonçait vouloir une PAC plus équitable.

Elle a ainsi proposé dans son paquet législatif d'octobre 2011 différents mécanismes de rééquilibrage des aides entre États-membres (EM) et entre exploitations agricoles bénéficiaires. Il y a eu beaucoup de mobilisation dans les différentes phases de consultation et de vote sur ces questions de la part des gouvernements, des parlementaires européens et des groupes d'intérêt. Cela a débouché sur des propositions alternatives, défendant le plus souvent des processus de convergence et de rééquilibrage plus progressifs, afin de limiter les écarts brutaux de revenu chez les bénéficiaires. L'accord de 2013 s'est donc fait sur un compromis très édulcoré par rapport

2. Commission européenne (CE) (2010). La PAC à l'horizon 2020 : alimentation, ressources naturelles et territoire. Relever les défis de l'avenir. Communication de la Commission au Parlement européen, au Conseil, au Comité économique et social européen et au Comité des régions, $16 \mathrm{p}$. 
au projet initial porté par la CE. Il laisse une grande marge de manœuvre aux EM pour infléchir dans un sens ou dans l'autre l'ambition redistributive qu'ils souhaitent donner à la réforme des paiements directs. L'année 2014 a été une année de transition au cours de laquelle s'est finalisée l'étape législative avec la traduction des actes de base en actes délégués et d'exécution et la préparation des mises en œuvre nationales et/ou régionales des nouveaux paiements directs. Début 2015, les États-membres ont fait connaître leurs décisions définitives sur les options qu'ils souhaitent activer pour la gestion des fonds alloués au premier pilier.

Les principales réformes du premier pilier ont consisté d'une part à rééquilibrer les soutiens de l'Union européenne attribués à chaque EM, et d'autre part à construire une nouvelle architecture des paiements directs afin de la rendre plus lisible et, dans les termes de la CE, plus « juste ».

Le premier processus dit de « convergence externe » a consisté à recalculer le montant des enveloppes budgétaires allouées à chaque EM pour les soutiens directs du premier pilier. L'objectif affiché est de doter chaque EM du même montant budgétaire par hectare de Surface Agricole Utile (SAU). Une règle pragmatique a été adoptée, fondée sur un principe de redistribution à somme nulle - les EM les mieux dotés devant financer l'augmentation de l'enveloppe des EM les moins dotés.

Le processus de « convergence interne » s'appuie sur une nouvelle structuration en strates des paiements directs. Tous les agriculteurs considérés comme « actifs » touchent un paiement de base par hectare de SAU, augmenté d'un paiement vert conditionné au respect de pratiques environnementales et qui doit représenter au moins $30 \%$ de l'enveloppe destinée aux paiements directs. Les montants du paiement de base par hectare, qui peuvent être calculés initialement à partir des montants des paiements uniques, doivent progressivement converger vers une valeur unique à l'échelle nationale ou régionale. Les EM doivent aussi proposer un paiement supplémentaire pour les jeunes agriculteurs qui s'installent (jusqu'à $2 \%$ de l'enveloppe). Par ailleurs, les EM peuvent s'ils le souhaitent prévoir, en sus de ces strates obligatoires, des paiements supplémentaires : pour les agriculteurs situés dans des zones à contraintes naturelles; pour les premiers hectares de chaque exploitation (appelé paiement redistributif) ; pour des productions vulnérables ou représentant un enjeu particulier (sous forme d'aides couplées).

La Commission européenne avait initialement proposé que les paiements de base soient plafonnés à $300000 €$ par exploitation. Après les négociations avec le Parlement et le Conseil, le plafonnement obligatoire a été abandonné. Il subsiste en revanche une dégressivité obligatoire, sous la forme d'un prélèvement de $5 \%$ minimum sur tous les paiements de base excédant $150000 €$ sauf dans les pays ayant choisi d'activer le paiement redistributif. Cette dégressivité peut être modulée en fonction du nombre de travailleurs de l'exploitation pour favoriser l'emploi.

Ces réformes peuvent-elles amener à une PAC plus juste ainsi que l'affirment les textes de la Commission européenne ? À partir d'indices d'inégalité, nous caractérisons dans un premier temps la concentration des aides directes avant la réforme (année 2013) à la fois entre États-membres, en fonction de leur SAU, et au sein des Étatsmembres, entre exploitations bénéficiaires, puisque ce sont ces deux critères qui ont guidé les procédures de convergence proposées par la CE. Nous n'abordons donc pas la question de la dispersion des aides agricoles entre les différents types de production ni entre les différentes classes de revenu, qui n'a pas fait partie du débat à Bruxelles, ou très peu. En revanche, nous analysons la façon dont la question 
de l'équité des aides a été traduite dans l'accord politique final, nous mesurons l'impact redistributif de la convergence externe, et nous présentons pour quelques États-membres, la France en particulier, comment ils choisissent d'utiliser la nouvelle architecture des aides pour faire évoluer la concentration des aides.

L'article rappelle dans une première partie les principaux mécanismes d'attribution des aides directes depuis 2003 et les critères de justice sociale qui peuvent être invoqués pour justifier les choix d'attribution des paiements aux agriculteurs. Dans une deuxième partie, il mesure la concentration des aides directes avant la réforme, entre États-membres et au sein des Étatsmembres. Dans la troisième et dernière partie, il décrit les principales dispositions de la réforme, analyse plus en détail les conséquences redistributives de la convergence externe et interne du paiement redistributif, et de la dégressivité.

\section{Les mécanismes d'attribution des aides directes depuis 2003 Quels critères d'équité ?}

\section{Le système d'attribution des aides directes après 2003}

Les agriculteurs européens reçoivent des aides dans le cadre de la PAC par : (i) des paiements directs de soutien au revenu, (ii) des mesures de marché (via l'Organisation commune de marché ${ }^{3}$ ), tous deux financés par le Fonds européen agricole de garantie (FEAGA) du premier pilier; et (iii) des aides au titre du développement rural cofinancées par les États-membres et le Fonds européen agricole pour le développement rural (FEADER), dans le cadre du deuxième pilier de la PAC.

3. Qui intervient pour la stabilisation des prix et la restructuration des marchés principalement dans les domaines du sucre, du lait, des fruits et légumes et du vin.
En 2012, les paiements directs représentaient plus de $90 \%$ du budget du premier pilier et plus des deux tiers du budget total annuel de la PAC. La Commission européenne estime également dans une analyse d'impact que, sur la période 2007 2009 , les paiements directs ont représenté en moyenne $29 \%$ du revenu agricole des agriculteurs européens (dépassant les $30 \%$ dans les États-membres de l'UE à 15 et atteignant presque $20 \%$ pour ceux de l'UE à 12 en moyenne) (CE, 2011b $)^{4}$. Il est donc légitime de s'intéresser à leur distribution.

Depuis la réforme à mi-parcours de 2003 et jusqu'à la fin de l'année de transition 2014, ces paiements sont versés directement aux agriculteurs dans le cadre du Régime de paiement unique (RPU) pour les anciens EM et du régime de paiement unique à la surface (RPUS) pour les nouveaux EM. Le calcul de ces paiements directs est fondé à l'échelle de chaque EM sur une enveloppe nationale, allouée par l'UE, qui leur est réservée et sur un nombre d'hectares admissibles. Les montants de ces enveloppes ont été calculés lors de la réforme à mi-parcours de 2003, en fonction des niveaux historiques de production des EM (CE, 2013c) $)^{5}$. Les montants reçus par les nouveaux EM (UE-12) ont été fixés quant à eux lors des accords d'adhésion à l'Union européenne et sont le produit d'une négociation politique et d'un calcul budgétaire qui ont conduit à accorder des montants moyens par hectare $30 \%$ plus bas que ceux pratiqués dans les anciens EM (UE-15). Les revendications croissantes pour un partage plus équitable du budget ont forcé l'Union européenne

4. Commission européenne (CE) (2011b). Impact assessment. Common Agricultural policy towards 2020. Annex 3, Commission staff working paper, $113 \mathrm{p}$.

5. Commission européenne (CE) (2013c). Report on the distribution of direct aids to agricultural producers (financial year 2012), 43 p. 
à reconsidérer cette question lors de la négociation de la PAC 2014.

Pour chaque exploitant agricole, la valeur des droits à paiement unique (DPU) qu'il touchait jusqu'en 2015 dépendait des modalités de distribution choisies par son pays. La majorité des anciens EM ont opté pour le modèle historique : chaque exploitant agricole a obtenu des DPU calculés sur la moyenne des aides qu'il a touchées entre 2000 et 2002. Pour les pays utilisant ce modèle, la distribution des paiements directs dépendait donc des choix de production faits par l'exploitant agricole durant ces années de référence. Deux exploitants identiques en termes de taille d'exploitations et de système de culture pouvaient donc toucher des DPU très différents en fonction de l'historique des aides perçues sur la période 2000-2002. D'autres EM ont mis en place un modèle mixte dans lequel ils pouvaient appliquer des systèmes de calcul différents d'une région à l'autre et pouvaient également déterminer les paiements en combinant aides historiques et taux forfaitaire ${ }^{6}$. C'est le cas de l'Allemagne, du Danemark ou encore de la Finlande. L'Angleterre, quant à elle, a poussé plus loin la logique de l'uniformisation en appliquant un taux unique de DPU/ha à l'échelle nationale. Enfin, les EM qui ont rejoint l'UE en 2004 et 2007 ont directement introduit un régime d'aide simplifiée liée à la surface et uniforme à l'échelle régionale.

Quel que soit le modèle de calcul des paiements uniques choisi, il reposait sur un système d'aides à la surface qui mécaniquement favorise les grandes exploitations. Il maintenait des disparités fortes entre les petites exploitations très peu aidées et les exploitations de grande taille qui cumulent ces aides à la surface (Cour

6. http://ec.europa.eu/agriculture/eval/reports/ decoupling/chapter3_fr.pdf des comptes européenne, 2011 et 2012)7. Le modèle historique, quant à lui, a maintenu des paiements élevés aux exploitations agricoles qui bénéficiaient déjà de soutiens forts au moment de la période de référence, lorsque les aides étaient versées quasi proportionnellement aux quantités produites et privilégiaient certains types de production.

C'est ce mode de distribution des aides qui est dénoncé comme injuste de façon récurrente par un certain nombre de parties prenantes : certains parlementaires européens, les syndicats agricoles minoritaires, mais aussi les associations de consommateurs et de défense de l'environnement.

\section{Quels critères de justice sociale derrière la logique de répartition des aides}

Lorsqu' on recense les critiques exprimées, on trouve globalement trois registres de revendications.

Le premier registre de critiques porte sur l'injustice globale des aides au secteur agricole par rapport aux autres secteurs de l'économie. Elles interrogent d'une part la légitimité d'un système qui verse chaque année près de 50 milliards d'euros d'aides européennes à un secteur d'activité qui ne répond pas toujours aux attentes de la société en termes de qualité alimentaire et environnementale, et d'autre part son efficacité puisque les budgets dédiés au secteur agricole se maintiennent malgré la baisse de l'importance de celui-ci dans l'économie et la diminution du nombre d'agriculteurs en Europe.

7. Cour des Comptes européenne (2011). Régime de paiement unique (RPU) : questions à examiner en vue d'améliorer la bonne gestion financière. Rapport spécial n ${ }^{\circ} 5,82$ p. Cour des Comptes européenne (2012). L'efficacité du régime de paiement unique à la surface en tant que système transitoire d'aide aux agriculteurs dans les nouveaux États-membres. Rapport spécial, $\mathrm{n}^{\circ} 16,58 \mathrm{p}$. 
Le deuxième type de critiques s'adresse plus spécifiquement à la répartition des aides. Il dénonce la concentration des aides et rappelle que, globalement, plus de $80 \%$ du montant total des aides distribuées sont captés par moins de $20 \%$ des exploitations. Une large partie des exploitations agricoles est donc exclue du système d'aides, qui ne bénéficie qu'à une minorité, alors que les budgets sont financés par l'ensemble de la collectivité.

Le dernier registre dénonce le fait que les aides vont majoritairement aux catégories d'agriculteurs qui en ont le moins besoin. Les céréaliers continuent de toucher des montants d'aide élevés alors même que leurs revenus se sont accrus fortement à la suite de la hausse des cours agricoles mondiaux, tandis que les producteurs de fruits et légumes qui font souvent face à des prix peu rémunérateurs reçoivent très peu d'aides directes au revenu. Certains think tanks ont ainsi défendu des règles d'attribution des aides qui mobiliseraient des critères très différents de ceux actuellement en vigueur et qui s'appuient beaucoup sur les surfaces cultivées. Ces critères pourraient être par exemple le besoin de revenu (sur le principe des aides sociales) ou un paiement par travailleur. La même critique existe à l'échelle des EM : la grande majorité des anciens États-membres bénéficie d'enveloppes d'aides (exprimées en €/ha de SAU ou en €/exploitation agricole) plus élevées que les nouveaux États-membres alors que ce sont ces derniers qui ont le plus besoin de moderniser leur secteur agricole.

Ces critiques font référence à des acceptions assez différentes de l'équité. Une première approche consiste à distinguer l'équité horizontale, qui stipule que deux personnes dans la même situation devraient avoir les mêmes droits et obligations, et l'équité verticale, qui s'appuie sur la réduction des écarts de niveau de vie entre individus. Alors que l'équité horizontale légitime plutôt des modes de distribution d'aide égalitaire fondés sur le principe « à circonstances égales, transferts égaux », l'équité verticale justifie des aides inégalitaires ciblées sur les exploitants en ayant le plus besoin. Pour autant, cette première distinction ne permet pas d'une part de définir ce qu'on appelle des « circonstances égales », et d'autre part, dans le contexte de l'équité verticale, les critères de répartition de l'effort de redistribution entre les individus.

Dans un article de 2002 consacré aux critères d'équité dans la répartition des aides directes aux agriculteurs, Butault et al. (2002) ${ }^{8}$ recensent trois manières d'évaluer l'équité d'une politique de transferts : on peut en effet considérer l'équité du processus d'allocation, l'équité du résultat obtenu par les transferts ou l'équité dans les critères d'attribution des transferts.

L'équité du processus d'allocation affirme que ce qui fait le caractère juste ou injuste d'une allocation n'est pas le résultat qu'elle atteint mais plutôt la façon dont cette allocation est décidée.

L'équité du résultat peut prendre plusieurs formes. Dans l'hypothèse où la société a une aversion très forte pour l'inégalité, le critère d'équité consisterait à calculer les aides de façon à lisser les inégalités de bien-être entre exploitants agricoles et à combler les différences de revenu avec les autres catégories socio-professionnelles dans l'économie. La version utilitariste au contraire consisterait à redistribuer les aides de façon à maximiser la somme des utilités dans la société, quitte, par exemple, à conditionner les aides aux contributions des agriculteurs à la fourniture de biens publics qui profitent à tous et augmentent le bien-être social.

8. Butault J.-P., Chantreuil F., Dupraz P., (2002). Critères d'équité et répartition des aides directes aux agriculteurs. Économie rurale, n ${ }^{\circ} 271$, pp. 84-91. 


\section{FAITS ET CHIFFRES}

Les notions d'équité fondées sur les critères d'attribution sont inspirées des courants post-welfaristes développés dans les années 1980. Sen avait introduit l'idée des capacities, c'est-à-dire de l'inégalité des individus dans leur capacité à transformer les biens en bien-être et du besoin pour ceux qui ont moins de « capacités » d'avoir accès à plus de ressources du fait de leur « handicap ${ }^{9}$. La question qui se pose alors dans ce constat d'inégalité est de distinguer ce qui est du ressort de la responsabilité de l'individu et ce qui est de l'ordre des « circonstances » indépendantes de sa volonté. Fleurbaey $(1996)^{10}$ distingue ainsi les ressources internes de l'individu qu'il ne peut contrôler ou maîtriser, comme ses talents intrinsèques, son origine familiale, sa position sociale, et tout simplement le facteur chance, de ses ressources internes contrôlables relevant de ses préférences, ses efforts et ses choix. Des critères d'attribution équitables des aides consisteraient à corriger les inégalités issues des premières en compensant davantage ceux qui ont de faibles ressources internes non contrôlables et en offrant le même niveau de transfert à ceux qui ont des talents ou handicaps équivalents (principe de compensation), mais à s'assurer aussi qu'à ressources contrôlables égales, les niveaux de réalisation ou de bien-être s'égalisent (principe de récompense naturelle).

Puisque la Commission européenne fait référence de façon répétée à une $\mathrm{PAC}$ « plus juste » dans sa justification de la réforme, il est intéressant d'analyser quels critères de justice sous-tendent la redistribution des aides proposée dans la réforme. En effet, ils ne sont jamais clairement exprimés.

Le rééquilibrage des montants moyens par hectare entre EM prévu dans le processus

9. Sen A. K. (1985). Commodities and Capabilities. Oxford India Paperbacks, Oxford University Press, $5^{\mathrm{e}}$ éd., 2000.

10. Fleurbaey M. (1996). Théories économiques de la justice. Paris, Economica. de convergence interne constitue avant tout un objectif politique. Il consiste à prélever sur les EM les mieux dotés pour transférer sur les EM moins dotés, tout en maintenant inchangée la moyenne européenne des aides à l'hectare. Ce choix n'est justifié ni par les «besoins » des EM ni par des soucis d'efficacité, mais par une volonté d'afficher un traitement purement égalitaire entre membres de l'Union.

La convergence interne est lourde de sens à deux titres : d'abord parce que la $\mathrm{CE}$, dans sa proposition de réforme de la PAC (CE, 2010) $)^{11}$, a réaffirmé le principe d'une aide surfacique et écarté d'autres options, par exemple le retour à des aides en fonction de la production, ou des paiements forfaitaires par exploitation ou par travailleur agricole, voire des aides versées en fonction du revenu. Le rééquilibrage est fondé avant tout sur la proposition que tout ha de SAU est éligible aux aides de base (moyennant des conditions d'entretien et le respect de certaines règles environnementales) et que ces aides à l'hectare doivent être identiques dans des régions agro-écologiques homogènes. La CE semble ainsi se positionner plutôt sur une logique d'équité horizontale fondée sur le foncier plutôt que sur les agriculteurs, qui stipulerait que deux hectares devraient avoir les mêmes droits d'accès aux aides pour des circonstances équivalentes. Un système de compensation des « handicaps », celui des aides supplémentaires versées aux surfaces situées en zones à contraintes naturelles, vient renforcer cette logique. Ce n'est qu'à travers l'aide aux jeunes agriculteurs (2\% seulement de l'enveloppe du $1^{\text {er }}$ pilier), l'aide optionnelle versée aux surfaces situées en zones à contraintes naturelles, et les aides du développement rural du $2^{\mathrm{e}}$ pilier (moins de $25 \%$ des budgets de la PAC) que l'on peut retrouver des critères d'attribution venant corriger les ressources internes non contrôlables et récompenser les « talents ».

11. Ibid. 
Par ailleurs, la CE affirme aussi son souci de garantir l'efficacité de la PAC, justifiant, dans une approche plus utilitariste, les dépenses publiques de la PAC par leur contribution à la croissance économique et à l'emploi. En rajoutant qu' « il s'agit donc de réfléchir à une répartition équitable qui réponde, de manière concrète et dans des conditions économiquement et politiquement réalisables, aux objectifs déclarés de ce soutien, mais également d'éviter tout changement brutal qui pourrait avoir de lourdes conséquences économiques et sociales pour certaines régions et/ou pour certains systèmes de production » $(\mathrm{CE}$, 2010) $)^{12}$, la CE rappelle aussi les contraintes de faisabilité politique d'une ré-allocation des aides qui pourrait s'apparenter à une remise en cause des droits acquis historiquement et être qualifiée aussi d'injuste dans une vision plus libertarienne et procédurale de l'équité.

Notons que la CE a aussi proposé de conditionner les aides à la contribution active des bénéficiaires au bien public environnemental (via le respect de pratiques respectueuses de l'environnement) et de limiter l'inégalité des aides entre exploitations agricoles en proposant un écrêtement (plafonnement et dégressivité) des aides directes allouées aux plus gros bénéficiaires, indépendamment de leurs « talents », de leurs « handicaps » et de leurs « droits acquis ». Elle s'inscrit là davantage dans une logique d'équité de résultats.

La multiplicité des critères d'équité auxquels on peut se référer accroît ainsi la tension entre EM et entre bénéficiaires (Dupraz et al., 2001 ; D’Oultremont, 2012) $)^{13}$. En effet, la conception d'une

12. Ibid.

13. Dupraz P., Leon Y., Pech M. (2001). Soutien public à l'agriculture et au développement rural : l'équité introuvable. Économie rurale, $\mathrm{n}^{\circ} 262$, pp. 109-116. D'Oultremont C. (2012). Introducing more equity in the CAP: a difficult challenge, $26 \mathrm{p}$. distribution équitable varie très largement d'un pays à l'autre. Alors que, pour certains EM, le fait que la plus grande part des soutiens bénéficient aux grandes exploitations n'est pas remis en cause, d'autres mettent en avant l'importance de ces aides pour des petites exploitations difficilement viables (Bureau et Witzke, 2010) ${ }^{14}$. Cela rend difficile la lisibilité des vertus du soutien et accentue les dissensions en période de disette budgétaire. Ce flou a certainement contribué à compliquer les négociations sur la réforme des soutiens et peut expliquer le choix d'une application « à la carte » pour permettre en partie aux EM de résoudre en interne les compromis sur l'équité qu'ils souhaitent faire.

\section{Caractérisation de la concentration des aides avant la réforme}

Pour mesurer les éventuels progrès en termes de redistribution des aides que la réforme de 2014 peut induire, il nous faut d'abord caractériser les inégalités dans l'allocation des aides avant 2014. Cette question est importante, car elle conditionne l'effort qui doit être fait : faut-il qu'il porte sur la convergence interne ou plutôt sur la convergence externe?

À l'échelle de l'UE, la principale source de données exhaustive et homogène sur la répartition des aides peut être trouvée dans les statistiques annuelles que publie la $\mathrm{CE}$ sur la distribution des aides du premier pilier par classes de tailles d'exploitation et par classes de montants reçus. Les statistiques de la CE différencient 10 tranches pour lesquelles on connaît le montant total d'aides touchées par les bénéficiaires de cette tranche et le nombre de bénéficiaires, sauf lorsqu'il y a moins de 10 bénéficiaires dans une tranche, ce qui explique que nos

14. Bureau J. C., Witzke H. P. (2010). The single payment scheme after 2013: new approachnew targets. Policy Department: Structural and Cohesion Policies, European Parliament, 173 p. 
calculs n'ont pu être faits que pour les $14 \mathrm{EM}^{15}$ présentant une série complète de données. Les dernières statistiques disponibles sont celles de $2013^{16}$ (CE, 2015) ${ }^{17}$. C'est donc cette source qui est principalement utilisée, ainsi que les données complémentaires spécifiques à chaque pays, gérées par Eurostat et la DG Agri, rassemblées chaque année dans les fiches techniques des $\mathrm{EM}^{18}$.

\section{Inégale répartition des aides entre ou au sein des États-membres ?}

L'analyse du problème de concentration des aides agricoles et les réponses proposées par la Commission européenne indique que les inégalités s'expriment à deux niveaux : entre les exploitationsbénéficiaires d'un même pays et entre les États-membres. Avant d'analyser plus en profondeur ces deux sources d'inégalité, il est intéressant de s'interroger sur leur poids respectif dans l'inégalité globale observée. Pour cela, nous avons utilisé un indice d'inégalité, l'indice de Theil T, classiquement défini de la manière suivante :

$$
T=\frac{1}{N} \sum_{i} \frac{R_{i}}{R} \times \frac{\log R_{i}}{R}
$$

Avec $\mathrm{N}$ le nombre d'individus, Ri l'aide attribuée à l'individu i et $\mathrm{R}$ l'aide moyenne attribuée à la population de $\mathrm{N}$ individus.

15. France, Lituanie, Pologne, Roumanie, Royaume-Uni, Allemagne, Danemark, Espagne, Italie, Hongrie, Portugal, République tchèque, Bulgarie, Slovaquie.

16. http://ec.europa.eu/agriculture/cap-funding/ beneficiaries/direct-aid/pdf/annex 1-2013_en.pdf

17. Commission européenne (CE) (2015). Indicative figures on the distribution of aid, by sizeclass of aid, received in the context of direct aid paid to the producers according to Council Regulation (EC). $N^{\circ} 1782 / 2003$ and Council Regulation (EC), $n^{\circ}$ 73/2009, financial year 2013, 28 p. (http:// ec.europa.eu/agriculture/cap-funding/beneficiaries/ direct-aid/pdf/annex1-2013_en.pdf)

18. http://ec.europa.eu/agriculture/statistics/ factsheets/pdf/eu_en.pdf
La borne inférieure de $\mathrm{T}$ est à 0 et indique une égalité parfaite. Plus T est élevé, plus la répartition des aides est inégalitaire. Cet indice présente l'avantage d'être décomposable et additif en sous-classes de bénéficiaires ce qui permet de distinguer la dispersion inter-classe de la dispersion intra-classe. Nous avons donc calculé l'indice de Theil global T pour les 14 EM dont l'ensemble des données sont disponibles sur l'année 2013 et nous l'avons décomposé pour pouvoir distinguer la part de la dispersion des aides liée à l'inégalité entre les pays $(\mathrm{Te})$ et celle liée aux inégalités à l'intérieur de chaque pays (Ti). $T=T_{e}+T_{i}$ Avec :

$$
\begin{gathered}
T_{i}=\sum_{g=1}^{14} \frac{N_{g}}{N} \times \frac{R_{g}}{R} T_{g} \\
T_{e}=\sum_{g=1}^{14} \frac{N_{g}}{N} \times \frac{R_{g}}{R} \times \log \frac{R_{g}}{R}
\end{gathered}
$$

Tg est l'indice de Theil ${ }^{19}$ du pays g, $\mathrm{Ng}$ l'effectif des exploitations agricoles bénéficiaires du pays $\mathrm{g}$, Rg le montant moyen d'aides dans le pays $g$, $\mathrm{R}$ et $\mathrm{N}$ sont respectivement le montant moyen d'aide et l'effectif global des exploitations agricoles bénéficiaires dans les $14 \mathrm{EM}$. Ti représente donc une moyenne pondérée des indices de Theil à l'intérieur de chaque pays et est donc un indicateur de la dispersion au sein des pays. En revanche, Te peut s'interpréter comme la valeur de l'indice de Theil si toutes les exploitations bénéficiaires dans chaque pays recevaient une aide égale à l'aide moyenne du pays. Te capture donc l'inégalité entre États-membres.

19. Dans notre cas, nous n'avons pas les aides individuelles, mais des montants et des nombres de bénéficiaires par classe. Nous construisons donc un individu «moyen » de la classe i dont l'aide Ri est le montant alloué à la classe divisé par le nombre de bénéficiaires de la classe, affecté d'un « poids » correspondant au nombre de bénéficiaires de la classe rapporté au nombre total de bénéficiaires dans le pays. 
Pour l'année 2013, l'indice de Theil est $\mathrm{T}=0,68$. La part de la dispersion intra-pays représente $70 \%$ de cet indicateur d'inégalité $(\mathrm{Ti}=0,47)$ tandis que la dispersion interpays ne pèse que pour $30 \%(\mathrm{Te}=0,21)$. Ces chiffres sont proches du même calcul appliqué aux données de 2010. Ce résultat indique que pour nos $14 \mathrm{EM}$, qui représentent $82,5 \%$ des montants distribués par l'UE, l'inégalité de distribution des aides agricoles repose beaucoup plus sur une inégalité entre exploitations bénéficiaires d'un même État-membre qu'entre Étatsmembres. Or une grande partie du débat sur le rééquilibrage des aides a porté sur la réallocation des enveloppes financières entre EM.

\section{Caractérisation de la dispersion des aides entre États-membres}

Dans la mesure où nous proposons des comparaisons entre pays, nous avons conduit notre analyse à partir de montants calculés en Parité de pouvoir d'achat (PPA), pour prendre en compte les différences de revenu et de niveaux de prix entre États-membres. Ce calcul nous permet donc d'étudier la question de la distribution des aides agricoles en lissant les différences entre les EM en termes de pouvoir d'achat des aides perçues par les agriculteurs. De fait, les aides du premier pilier sont des aides dites de « soutien au revenu » dont une partie est donc utilisée pour la consommation des ménages agricoles. Cette correction est rarement faite dans les analyses existantes alors que, malgré le processus d'intégration, la différence de pouvoir d'achat de l'euro reste encore grande. Par exemple, ce taux de conversion, calculé par rapport à la moyenne de l'UE-27, peut varier pour les pays membres de la zone euro de 0,77 pour le Portugal à 1,21 pour le Luxembourg.

Lorsqu' on compare le montant des aides directes (en PPA) par hectare de surfaces agricoles potentiellement éligibles (PEA) ${ }^{20}$ dans l'UE-27 en 2013, on peut observer un écart de 1 à 6 entre les pays les plus dotés et les pays les moins dotés (les données sont rassemblées en annexe 2). Mais la courbe de Lorenz présentée dans le graphique 1, qui montre que $60 \%$ des hectares agricoles européens cumulent $50 \%$ de montant des aides directes, relativise cette première impression. L'indice de Gini en parité de pouvoir d'achat est $\mathrm{G}_{(\mathrm{ha})}=0,138$. Si l'on reste sur les montants nominaux sans corriger pour les différences de niveau de prix, il est un peu plus élevé $\mathrm{G}^{*}{ }_{\text {(ha) }}=0,171$ mais reste bas. La répartition des montants d'aides directes en fonction des hectares de surfaces potentiellement éligibles est donc finalement peu inégalitaire.

\section{Encadré 1. Construction des courbes de Lorenz et calcul de l'indice de Gini}

La courbe de Lorenz, dans notre cas d'étude associe à chaque part de la population ordonnée par montant d'aide touchée croissant (aides directes/ha de surfaces potentiellement éligibles dans le graphique 1 et aides directes/bénéficiaires), la part que représente les aides qu'elle touche dans le montant total des aides distribuées. L'indice de Gini se mesure en rapportant le double de la différence entre l'aire délimitée par la courbe de Lorenz et la bissectrice, et l'aire totale sous la bissectrice. L'indice de Gini donne une mesure de l'inégalité de la distribution des aides directes entre États-membres. Un indice de Gini égal à 1 traduit une répartition parfaitement « inégalitaire »: tous les pays membres de l'Union sauf un ne toucheraient aucune aide. Un indice de Gini égal à 0 traduit en revanche une répartition parfaitement « égalitaire » au sens où chaque Étatmembre toucherait un pourcentage d'aides reflétant exactement son poids (en hectare de PEA ou en nombre d'exploitations bénéficiaires) dans l'UE.

20. Ce sont les surfaces agricoles nationales de référence à partir desquelles sont calculées les enveloppes budgétaires du premier pilier pour chaque EM. 
Graphique 1. Courbe de Lorenz du montant moyen d'aides directes par hectare de surfaces potentiellement éligibles en parité de pouvoir d'achat dans I'UE-27 (données 2013) - classement des États-membres en fonction du montant d'aides par hectare de PEA

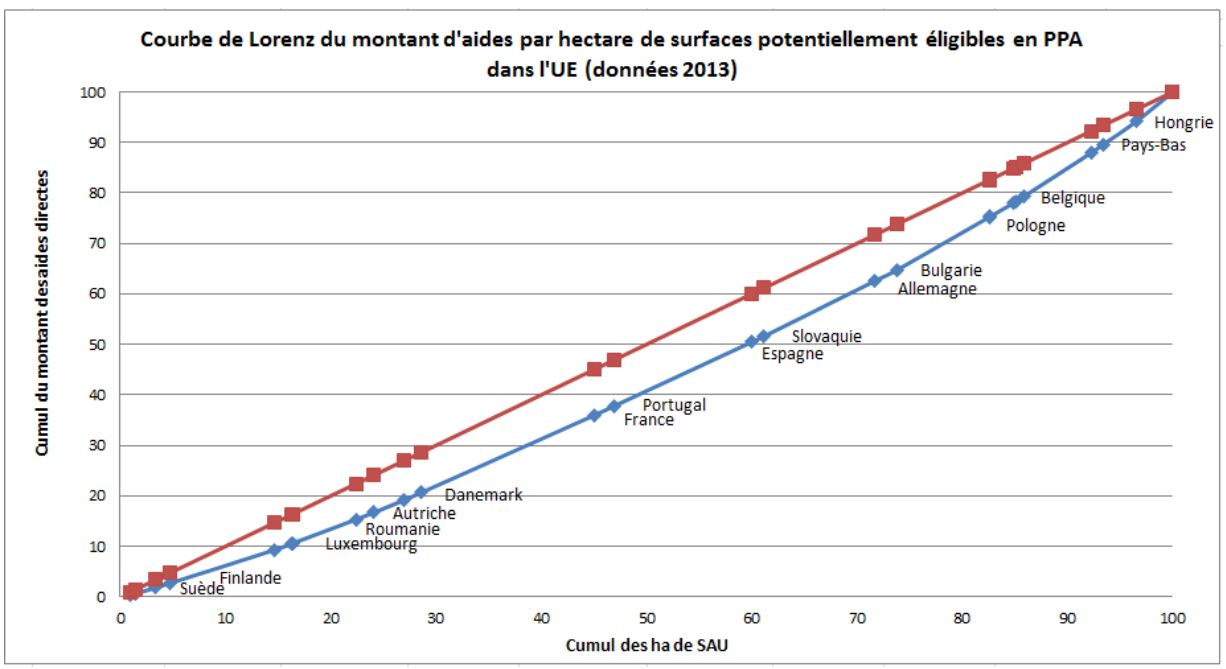

Source : les auteures.

Les écarts entre EM sont plus importants lorsqu'on considère les montants moyens d'aides directes par Exploitation agricole (EA) bénéficiaire, reflétant ainsi l'hétérogénéité des tailles moyennes des exploitations entre EM. Le montant moyen européen d'aides directes par EA est de $5628 €$ (en PPA). C'est en République tchèque que le montant moyen par exploitation agricole est le plus élevé (42 $567 €$, la taille moyenne des EA est de 152 ha de SAU) et à Malte qu'il est le plus faible (1 $044 €$, la taille moyenne des EA maltaise est de 0,9 ha de SAU) (graphique 2).

À l'échelle de l'UE, $51 \%$ des bénéficiaires les moins aidés ne cumulent que $24 \%$ du montant total des aides directes, tandis que les $8 \%$ de bénéficiaires touchant le plus se partagent $25 \%$ des aides directes. L'indice de Gini en PPA est $\mathrm{G}_{\text {(bénéf) }}=0,411$.

Il est également important de noter ici la part des bénéficiaires sur la totalité des exploitations agricoles. En effet, pour certains États-membres cette part est très faible : c'est le cas de la Hongrie, de la Roumanie ou encore de la Bulgarie où le nombre de bénéficiaires représente $30 \%$ ou moins du nombre total d'EA du pays (disponibles pour tous les États-membres en annexe 2). Sans surprise, l'indice de Gini en PPA lorsque l'on prend en compte l'ensemble des exploitations est encore plus élevé, $\mathrm{G}_{(\mathrm{ea})}=0,552$ (en PPA).

\section{Dispersion des aides au sein des États-membres Une France finalement peu inégalitaire}

Comme l'a montré le calcul de l'indice de Theil à l'échelle de l'UE, une grande partie de l'inégale distribution des aides directes est liée à leur distribution au sein des Étatsmembres. Nous avons pour ces mêmes 14 EM déterminé les indices de Gini et de Theil à l'échelle nationale afin de caractériser cette dispersion et tenter de voir dans quelles mesures les modèles d'attribution des aides impactent l'inégalité de distribution au sein des États-membres. 
Graphique 2. Écarts à la moyenne du montant moyen d'aides directes par bénéficiaire en parité de pouvoir d'achat (données 2013)

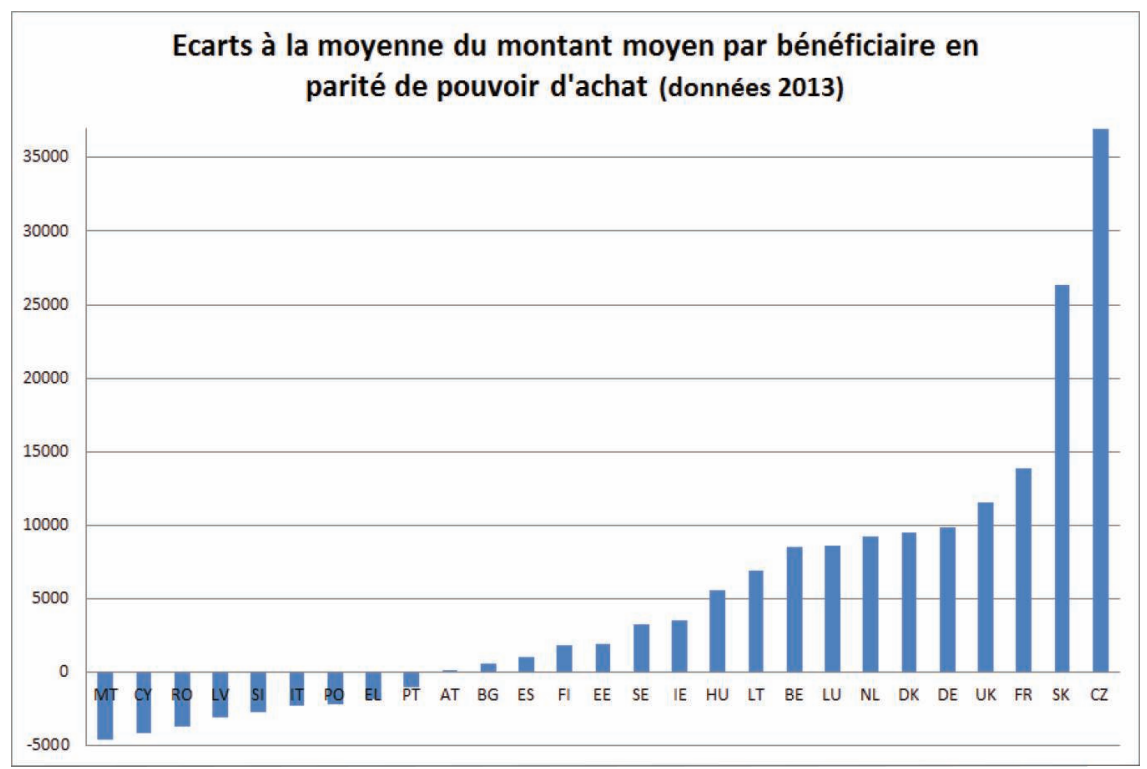

Source : les auteures.

L'indice de Theil est plus sensible que l'indice de Gini aux inégalités dans les parties extrêmes de la distribution, ce qui explique les différences de classement induites par ces deux indices (graphique 3). Si la France reste, sur les 14 pays étudiés, celui qui est le moins inégalitaire en termes de répartition des aides, la Roumanie régresse de la $6^{\mathrm{e}}$ place à la $13^{\mathrm{e}}$ place avec l'indice de Theil qui sanctionne ainsi une distribution caractérisée par de nombreux bénéficiaires de très petits montants et de gros montants cumulés sur quelques grandes exploitations.

On remarque que l'on retrouve d'avantage de nouveaux États-membres (modèle RPUS qui prévoit l'octroi à tous les agriculteurs d'un montant forfaitaire) en fin de classement aussi bien pour l'indice de Gini que pour celui de Theil (Bulgarie, Slovaquie, Hongrie et République tchèque), ce qui montre bien le poids des différences structurelles existantes dans ces pays où, comme dans le cas de la Roumanie, de très petites exploitations agricoles en côtoient de très grandes.

Cependant, et contrairement à l'intuition, un lien clair entre les indices d'inégalité et les modèles de mise en œuvre de distribution des aides directes adoptés par les EM semble difficile à mettre en avant. Ainsi, la France qui a opté pour un modèle historique est moins inégalitaire que l'Allemagne ou le Danemark qui ont pourtant adopté un modèle mixte.

Compte tenu de ce premier panorama, que peut-on dire sur les ambitions de la réforme de 2014 ?

\section{La réforme de 2014 : quelques effets redistributifs attendus... ou évités}

En cherchant à limiter la redistribution potentielle dans un souci de pragmatisme 
Graphique 3. Résultats des indices de Gini et de Theil de différents États-membres de l'UE-27
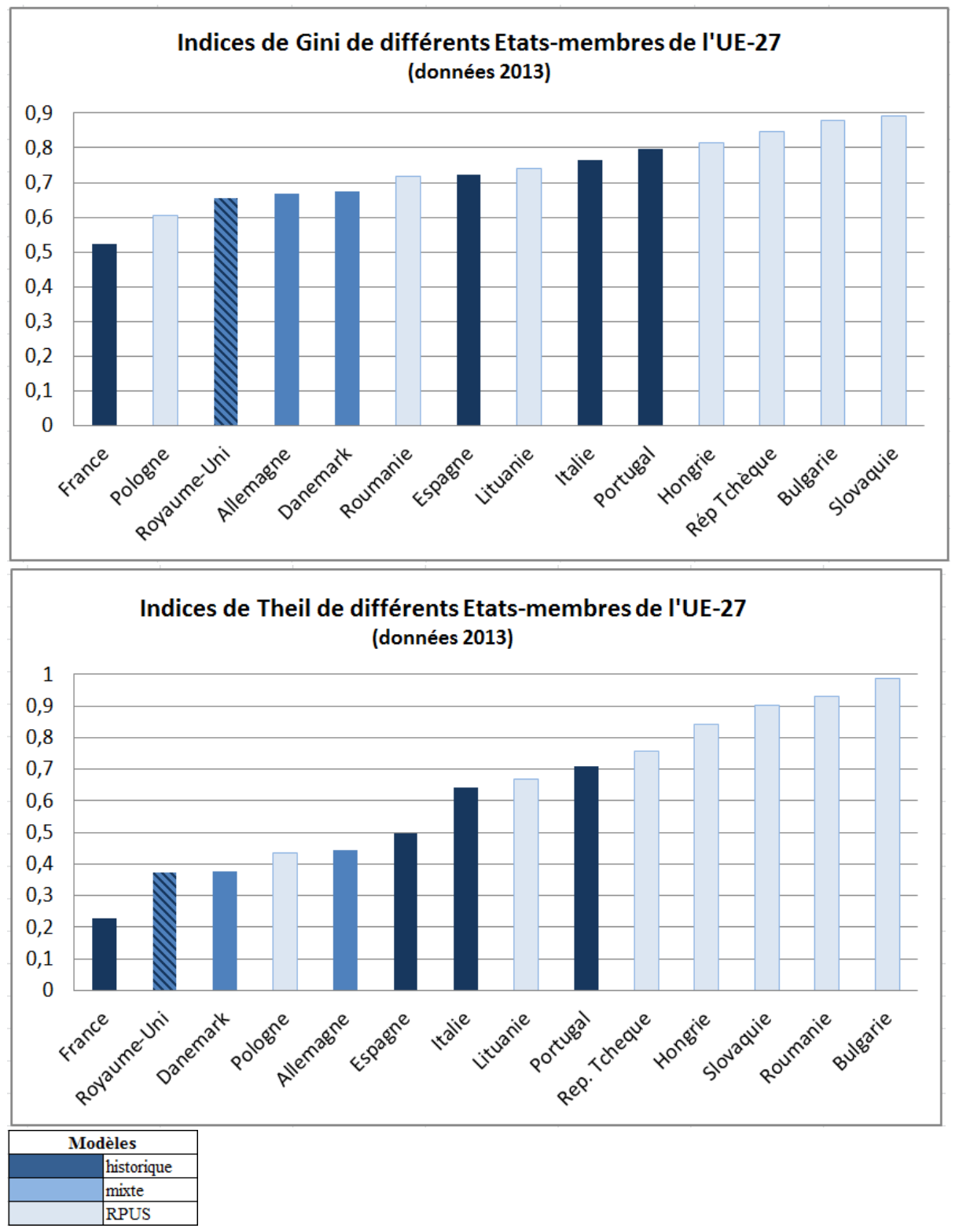

Source : les auteures. 
politique, l'accord obtenu entre le Conseil, le Parlement et la Commission s'est éloigné de la logique de départ de la Commission (Adinolfi et al., 2011) ${ }^{21}$. Il s'inscrit davantage dans un critère d'équité de consensus correspondant plus à une logique de marchandage qu'à un concept théorique donné (Butault et al., 2002)22 et révèle ainsi qu'au-delà d'une aversion commune pour l'inégalité, aucun consensus n'émerge réellement sur les motivations sous-jacentes.

\section{La convergence externe, une mesure à impacts limités}

Pour répondre à l'enjeu d'une répartition plus équitable des aides directes entre les EM, la CE a proposé une convergence des montants moyens d'aides directes par hectare éligible. Dans sa proposition qui a servi de base à la négociation, la $\mathrm{CE}$ a tenté d'estimer le degré de redistribution politiquement acceptable à la fois par les Étatsmembres dont les budgets seront réduits et par ceux qui verront les leurs augmenter, et a donc simulé plusieurs scénarios redistributifs (Swinbank, 2012) ${ }^{23}$.

L'accord politique obtenu précise que les EM dont la moyenne des paiements à l'hectare est inférieure à $90 \%$ de la moyenne européenne doivent bénéficier d'une hausse progressive de leur enveloppe de façon à combler au moins un tiers de cet écart. Cette convergence est financée

21. Adinolfi F. Little J., Massot A. (2011). La PAC à l'horizon 2020 : scénarios envisageables pour la redistribution $d u$ budget des paiements directs. Note, Parlement européen, Direction générale, Politiques internes de l'Union, Département thématique $\mathrm{B}$, Politiques structurelles de Cohésion agriculture et Développement rural, $42 \mathrm{p}$.

22. Ibid.

23. Swinbank A. (2012). New direct payments scheme: targeting and redistribution in the future $C A P$. Note, Parlement européen, Direction générale, Politiques internes de l'Union, Département thématique $\mathrm{B}$, Politiques structurelles de Cohésion Agriculture et Développement rural, 74 p. proportionnellement par tous les EM dont le niveau des paiements directs est supérieur à la moyenne de l'Union. L'accord obtenu avec le Parlement et le Conseil a retenu cette proposition en y ajoutant une condition supplémentaire sur un rattrapage plancher pour les pays les moins dotés, imposant que le niveau d'aides directes par hectare atteigne au minimum $196 €$ d'ici 2020.

À partir des surfaces potentiellement éligibles (PEA) de chaque État-membre et des montants plafonds prévus dans le nouveau règlement de la $\mathrm{PAC}^{24}$, nous avons donc déterminé le montant moyen d'aides directes par EM en 2019 après la mise en œuvre de la convergence externe. Nous proposons ici de le comparer au niveau des montants moyens de 2013 hors PPA (graphique 4). Nous constatons d'abord que ce calcul donne des résultats sensiblement différents des simulations présentées par la CE en 2012 (Swinbank, 2012) (25 $^{25}$ et reprises ensuite dans la plupart des documents de présentation de la réforme.

On observe en effet qu'entre 2013 et 2019, les montants moyens d'aides par hectare de PEA diminuent pour 14 EM, y compris pour certains qui se situaient en 2013 en dessous des $90 \%$ de la moyenne européenne ( $257 € /$ ha). Cela s'explique en partie par le fait que les enveloppes financières de 2019 intègrent non seulement la convergence externe mais aussi la diminution globale du budget de la $\mathrm{PAC}^{26}$, ce qui fait baisser mécaniquement la moyenne de référence. Certains cas particuliers, comme Chypre, s'expliquent par des enveloppes négociées au cas par cas.

24. Règlement (UE) $n^{\circ} 1307 / 2013$ du Parlement européen et du Conseil du 17 décembre 2013 établissant les règles relatives aux paiements directs en faveur des agriculteurs au titre des régimes de soutien relevant de la politique agricole commune en abrogeant le règlement $(\mathrm{CE}) \mathrm{n}^{\circ} 637 / 2008 \mathrm{du}$ Conseil et le règlement $\mathrm{n}^{\circ} 73 / 2009 \mathrm{du}$ Conseil.

25. Ibid.

26. Diminution d'environ 3,5\% par rapport à la programmation 2007-2013. 
Graphique 4. Montants d'aides directes moyens par hectare de surfaces potentiellement éligibles hors PPA en 2013 et estimations d'après les plafonds 2019

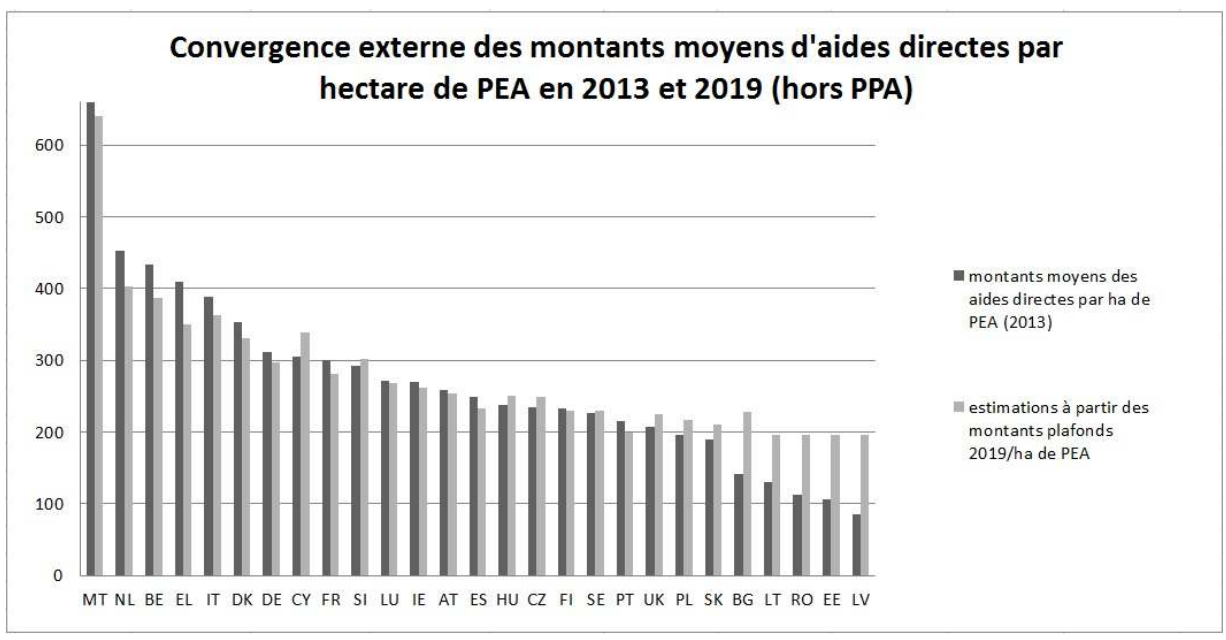

Note : Code des pays : voir annexe 1

Source : les auteures.

Pour estimer le montant transféré par les EM contributeurs en ne prenant en compte que la convergence, nous avons utilisé les montants moyens par hectare de PEA de 2013, en leur administrant les règles de calcul décidées à la suite de l'accord de juin 2013. Ainsi, on estime que le montant à transférer via la convergence est d'environ 1795 millions $€$. Les EM contributeurs dédieront donc en moyenne moins de $8 \%$ de l'ensemble de leur enveloppe nationale 2013 à la convergence. Pour les pays dont le montant était inférieur à $90 \%$ de la moyenne, la plus forte augmentation concerne la Lettonie, l'Estonie et la Roumanie qui voient leur montant/ha atteindre $196 €$ (contre respectivement $86 € /$ ha, $106 € /$ ha et $112 € /$ ha sans convergence externe). En ne prenant en compte que les effets de la convergence externe, sans la réduction budgétaire, on atteint un indice de Gini de $\mathrm{G}^{*}{ }_{\text {(conv) }}=0,104$ (hors PPA) en baisse de $39 \%$ par rapport à celui de 2013.
La convergence externe a donc soulevé de nombreux débats, mais elle reste très limitée en montant redistribué $(4,3 \%$ du montant total européen des aides directes) et ne permet qu'un rattrapage partiel des EM les moins dotés. Le montant plancher de 196 €/ha ne représente que $76 \%$ de la moyenne européenne alors que certains EM (Malte, les Pays-Bas ou la Belgique) conserveront des montants moyens par hectare après convergence très élevés, représentant le double voire plus, de ce plancher. Par ailleurs, certains futurs bénéficiaires de la convergence externe sont des EM dont les montants moyens d'aide par exploitation agricole sont les plus élevés de l'UE, comme la Slovaquie. Il faut aussi noter, hasard ou pas, que dans l'analyse d'impact de la Commission européenne, le scénario se rapprochant le plus de la mesure finalement négociée était celui dont l'impact sur le revenu agricole des agriculteurs était le plus faible : peu d'augmentation de revenu pour les nouveaux EM 
$(+2 \%)$ et moins de $-1 \%$ de diminution pour l'UE à $15^{27}$ (CE, 2011b) ${ }^{28}$.

Il semble bien que le pragmatisme politique et les contraintes d'acceptabilité sociale aient pesé plus lourd que la volonté de rééquilibrer les aides entre EM en fonction de leurs besoins ou d'une stratégie agricole intégrée à l'échelle européenne. Les sommes redistribuées sont faibles mais une grande publicité a été faite, masquant un peu une ambition initiale très écornée.

\section{Les modalités de la convergence interne des aides agricoles}

En 2015, tous les EM aujourd'hui dans le régime de paiement unique passent au nouveau système des Droits à paiement de base (DPB) versés à l'hectare. La différence avec le système actuel des paiements uniques est que le paiement de base sera versé à tous les agriculteurs définis comme « agriculteurs actifs » ${ }^{29}$ qui non seulement sont en conformité avec les règles de la conditionnalité mais qui respectent aussi des mesures additionnelles dites de « verdissement ». Ce paiement de base s'applique à toutes les terres agricoles dites « admissibles $»^{30}$. Ce nouveau système

27. Le scénario du paiement forfaitaire unique dans l'UE indique $+8,6 \%$ du revenu agricole dans 1'UE-12 et $-2,1 \%$ pour l'UE- 15 .

28. Ibid.

29. La notion d'agriculteur actif est définie dans un premier temps au niveau européen par l'exclusion de certains bénéficiaires comme les aéroports ou les compagnies ferroviaires par exemple. Cette notion sera précisée à l'échelle de chaque Étatmembre dans un second temps.

30. La notion d' « hectare admissible » a été définie par la CE comme étant « toute surface agricole de l'exploitation utilisée aux fins d'une activité agricole ou, lorsque la surface est utilisée également aux fins d'activités non agricoles, essentiellement utilisée à des fins agricoles ». Les EM ont cependant une certaine marge de manœuvre sur l'inclusion dans ces surfaces admissibles des surfaces qui bénéficient des soutiens de l'OCM unique (par exemple les surfaces viticoles ou les vergers). vise clairement à rompre avec la logique de la référence historique (CE, 2011a $)^{31}$.

Après de longues négociations avec le Parlement et le Conseil, le concept de convergence interne adopté est le suivant : il faudra qu'au sein d'un même État-membre ou d'une région, l'aide à l'hectare soit au moins égale à $60 \%$ de la moyenne des aides nationales/régionales d'ici 2019 et que les agriculteurs avec des DPB d'une valeur inférieure à $90 \%$ de la moyenne régionale ou nationale comblent au moins $30 \%$ de leur écart à la moyenne. Les montants mis à la disposition des agriculteurs qui reçoivent plus de la moyenne régionale/nationale seront ajustés en proportion, mais les EM auront la possibilité de limiter la réduction des DPB en 2019 à $30 \%$ de leur valeur initiale de 2015 (CE, 2013b) ${ }^{32}$ afin d'éviter une trop forte baisse pour les agriculteurs qui bénéficiaient jusqu'à présent, le plus souvent dans le cadre du régime de références historiques, des aides les plus élevées.

L'Allemagne et l'Angleterre, qui avaient adopté lors de la réforme à mi-parcours des modèles proches de ce qui est prévu dans la réforme actuelle, ont opté pour une convergence totale. C'est aussi le cas pour les Pays-Bas qui jusque-là avaient suivi un modèle historique. Pour les 12 nouveaux EM, la date d'expiration du système fondé sur le régime de paiement unique à la surface sera prolongée jusqu'en 2020. Le compromis obtenu impose donc aux EM d'engager le processus de convergence, mais les dispositions retenues peuvent leur permettre de rester encore relativement loin de l'objectif d'un paiement à l'hectare forfaitaire national/régional.

31. Commission européenne (CE) (2011a). Proposition de règlement du Parlement européen et du Conseil établissant les règles relatives aux paiements directs en faveur des agriculteurs au titre des régimes de soutien relevant de la Politique agricole commune, $81 \mathrm{p}$.

32. Commission européenne (CE) (2013b). Réforme de la PAC, explications des principaux éléments. Mémo, 9 p. 
En France, la sortie des références historiques sera progressive. En effet, elles seront utilisées pour déterminer la valeur initiale théorique des DPB : le ratio DPU de l'agriculteur/DPU moyen français de 2014 sera conservé dans le rapport entre les nouveaux DPB attribués à l'agriculteur et le DPB moyen français. La convergence sera déterminée en fonction de cette valeur initiale, les agriculteurs ayant des DPB supérieurs à la moyenne nationale financeront une augmentation des DPB chez les agriculteurs ayant des DPB inférieurs à la moyenne nationale. Notons que la France, en accord avec la profession agricole, a choisi de sortir ses surfaces en vignes des surfaces admissibles aux DPB.

L'objectif que s'est donné la France est que tous les DPB dont le montant est inférieur à la moyenne nationale voient leur valeur augmenter pour atteindre $70 \%$ de la moyenne nationale en 2019 (qui sera d'environ $93 €)^{33}$. Le mécanisme de limitation des pertes sur les droits à paiement de base, autorisé par la CE pour réduire l'impact de la convergence sur les détenteurs de DPB élevés, est aussi activé par la France : les réductions des montants des DPB seront limitées à $30 \%$ de la valeur initiale de référence. La convergence sera donc réelle mais étalée sur 4 années et non totale. La France a choisi d'utiliser les mécanismes permettant de limiter l'impact sur les EA touchant historiquement le plus d'aides directes. En revanche, la France a défendu avec beaucoup de conviction, et obtenu, l'inclusion dans le Règlement de la possibilité de mettre en place un paiement redistributif destiné à « supprimer » les premiers hectares de chaque exploitation.

33. En 2019, en France l'enveloppe nationale française sera d'environ 7,19 milliards d'euros et sera partagée entre : $15 \%$ pour les aides couplées, $1 \%$ d'aides pour les jeunes agriculteurs, $20 \%$ pour la surprime, $30 \%$ pour le verdissement et le reste soit $34 \%$ pour les droits à paiements de base.

\section{Le paiement redistributif aux premiers hectares. La bonne solution ?}

Malgré la position largement majoritaire des EM en faveur d'une limitation de la convergence interne, le Conseil, sous impulsion française, qui a pourtant l'index de Gini le plus bas de nos 14 EM étudiés, a soutenu l'ajout facultatif d'une surprime pour les premiers hectares. Elle vise une redistribution vers les exploitations supposément « riches en emploi », qui, pour le cas français, se trouvent être plutôt des petites et moyennes exploitations (ministère de l'Agriculture, de l'Agroalimentaire et de la Forêt, 2013) $)^{34}$. Le Règlement européen stipule que la surprime des premiers hectares est limitée aux 30 premiers hectares de chaque exploitation ou à la SAU moyenne de l'EM si elle est plus élevée que 30 ha. Elle peut être prise en charge à hauteur maximale de $30 \%$ du plafond national et le montant de la surprime sera calculé en augmentant de $65 \%$ au maximum la valeur moyenne du droit de paiement de base national ou régional. En contrepartie, les EM choisissant d'activer la surprime peuvent ne pas appliquer la dégressivité. Ce système contribue certes à réduire la concentration des aides, mais il permet aussi que l'effort de redistribution soit plus largement réparti sur l'ensemble des exploitations au-dessus de la taille moyenne nationale tout en épargnant aux plus grandes les prélèvements liés à la dégressivité (Matthews, 2013) ${ }^{35}$. L'efficacité de cette mesure repose malgré tout sur le choix des EM de la mettre en place et sur la part de l'enveloppe nationale qu'ils lui dédient.

34. Ministère de l'Agriculture, de l'Agroalimentaire et de la Forêt (2013). PAC 2014/2020 : Comment assurer une redistribution en faveur de l'élevage et de l'emploi ? $22 \mathrm{p}$.

35. Matthews A. (2013). Implications of the new redistributive payment, blog Capreform. http:// capreform.eu/implications-of-the-new-redistributive-payment/ 
L'Allemagne et la France, ainsi que la Roumanie, la Lituanie, la Bulgarie, la Pologne, la Hongrie et la Wallonie ont décidé de mettre en place la surprime (voir annexe 2). En Allemagne, il a été décidé d'attribuer aux 15 premiers hectares $50 € /$ ha et $30 € /$ ha pour les 15 suivants, évitant ainsi de mettre en place la dégressivité. L'ancienne ministre de l'Agriculture allemande a mis en avant l'importance d'éviter de trop pénaliser les exploitations allemandes de l'Est devant déjà subir la diminution du budget du $1^{\text {er }}$ pilier allemand ${ }^{36}$.

Dans le cas français, le seuil de surprime des premiers hectares est placé à la moyenne de la SAU des exploitations agricoles, 52 ha. Cela concerne environ 14,5 millions d'ha, soit plus de $55 \%$ des surfaces éligibles. La redistribution des aides va profiter entre autres à l'Otex élevage (+12\% d'aides par rapport à 2010 - ministère de l'Agriculture, de l'Agroalimentaire et de la Forêt, 2013) ${ }^{37}$. La surprime représentera $5 \%$ de l'enveloppe nationale française dès 2015 , et il est prévu de l'augmenter pour qu'elle atteigne $20 \%$ en $2019^{38}$. En prenant en compte la diminution de l'enveloppe nationale, le montant moyen de la surprime passera en moyenne de $26 € /$ ha en 2015 à $103 € /$ ha en 2019. En France, le choix du paiement redistributif a été justifié par une volonté de soutien du secteur de l'élevage ${ }^{39}$. Les céréaliers français s'inquiètent d'ailleurs de cette redistribution : les résultats de leurs

36. http://www.bmelv.de/SharedDocs/ Pressemitteilungen/2013/SprechererklaerungMeyer-zu-Umsetzung-der-GAP.html

37. Ibid.

38. Une évaluation du dispositif de surprime sera réalisée en 2016 pour éventuellement revoir l'objectif de $20 \%$ de l'enveloppe nationale française prévue pour la surprime en 2019.

39. À noter également que la France a choisi d'utiliser le budget maximal possible pour les aides couplées et que l'élevage constitue une priorité : $59 \%$ de l'enveloppe des aides couplées concerne les vaches allaitantes, et $11 \%$ les ovins. propres simulations montrent une diminution importante des aides moyennes en grandes cultures, selon eux sous-estimée dans l'étude du ministère de l'Agriculture français. Ils estiment que cela risque de les pénaliser face à leurs homologues européens soumis à des conditions de redistribution moins fortes (AGPB, 2013 ; Orama, $2013)^{40}$.

\section{De faibles montants redistribués via la dégressivité}

La CE avait souligné « l'octroi disproportionné des paiements directs à un nombre relativement réduit de gros bénéficiaires ». En effet, elle estime que ceux-ci bénéficient d'économies d'échelles et « n'ont pas besoin, aux fins de l'objectif du soutien au revenu, du même niveau de soutien ». « En outre, leur potentiel d'adaptation leur permet plus facilement de fonctionner avec des niveaux de soutien moindres » $(\mathrm{CE}, 2011 \mathrm{a})^{41}$. À l'échelle de l'Union européenne, en 2012 seulement $0,2 \%$ des bénéficiaires des paiements directs touchaient plus de $150000 €$ d'aides et cumulaient $11 \%$ du montant total des aides directes.

Suite à l'accord du 26 septembre 2013, il a été décidé de rendre facultatif le plafonnement des aides directes, mais de maintenir obligatoire une dégressivité du montant des paiements de base au-delà de $150000 €$ et à hauteur minimale de $5 \%$. Afin d'éviter des effets disproportionnés sur les grandes exploitations dont les effectifs de salariés sont importants et pour préserver l'emploi agricole, les coûts salariés

40. Association générale des producteurs de blé et autres céréales (AGPB) (2013). Paiements/ hectare "nouvelle PAC»: scénarios du ministère. Commentaires de l'AGPB, communiqué de presse publié le 25 juillet 2013. Orama (2013). Application en France de la nouvelle PAC : il est impératif de mettre en auvre une solution équilibrée. Communiqué de presse publié le 6 septembre 2013.

41. Ibid. 
pourront être déduits. Les fonds « récupérés » resteront dans l'État-membre ou la région concerné(e) et pourront être utilisés dans le cadre du second pilier de la PAC sans obligation de cofinancements.

Seize des vingt-huit États-membres ont choisi de mettre en place le minimum autorisé, c'est-à-dire une dégressivité de $5 \%$ pour les montants supérieurs à $150000 €$ (voir annexe 2). Alors que certains EM ajoutent à la dégressivité, un plafonnement à un montant supérieur à $150000 €$ (c'est le cas par exemple de l'Italie ou de la Hongrie), trois EM seulement, l'Irlande, la Grèce et la Belgique, ont opté pour un plafonnement dès $150000 €$.

Une estimation du nombre de bénéficiaires et des montants concernés dans les États-membres est difficile à ce stade de la mise en œuvre des droits à paiement de base dans chaque EM étant donné que seuls les DPB seront soumis à dégressivité. En effet, même si la part de l'enveloppe nationale qui sera dédiée aux paiements de base a été fixée ${ }^{42}$ par les États-membres, la diffusion par tranches de la répartition de ces montants ne nous permet de faire qu'une estimation approximative des montants qui pourront ainsi être prélevés par la dégressivité et le plafonnement. Nous l'estimons, à partir des données disponibles de 2013, à 310 millions $€$, soit environ $0,7 \%$ de l'enveloppe totale des aides directes de l'UE-27 en 2013. C'est l'Italie avec un taux de dégressivité de $50 \%$ pour les montants de droits à paiement de base supérieurs à $150000 €$ et un plafond de $500000 €$ qui participe le plus largement à cette enveloppe.

$*$
$* *$

La Commission européenne dans sa proposition de paquet législatif pour la

42. Elle dépend des enveloppes dédiées aux jeunes agriculteurs, aux aides couplées, aux zones défavorisées.
PAC 2014-2020 souhaitait introduire plus d'équité dans la distribution des aides agricoles entre États-membres et au sein des États-membres. Cependant, l'analyse des mesures proposées montre que l'ampleur de cette redistribution restera finalement très modérée, du moins pour les Étatsmembres qui ne souhaitent pas actionner les dispositifs permettant de redistribuer davantage.

La convergence externe a l'avantage de faire financer le rattrapage des EM dont le montant moyen d'aides par hectare est faible par ceux pour lesquels il est plus élevé. Mais l'intensité du "rattrapage » reste modeste et n'affectera qu'à la marge les disparités entre États-membres, qui s'expriment davantage dans leurs capacités à cofinancer les aides du deuxième pilier.

Dans l'inégale répartition des aides, ce sont principalement les différences entre bénéficiaires qui pèsent. La réforme de la PAC met fin aux références historiques, de façon progressive, et donne l'opportunité aux EM de s'orienter vers une distribution plus équitable via la convergence interne, la dégressivité et le paiement redistributif. Elle laisse cependant de grandes marges de manœuvre dans l'application des mesures.

Bien que l'efficacité des outils proposés par la Commission européenne pour une meilleure redistribution des aides agricoles semble faible, la réforme a néanmoins le mérite de poser différemment la logique de l'allocation des aides et donc d'entrouvrir la porte à des redistributions plus ambitieuses à l'avenir. En revanche, la très (trop ?) grande flexibilité laissée aux États-membres pour gérer la redistribution risque d'accentuer encore les distorsions de concurrence entre exploitations européennes, déjà fortes du fait des différences de politiques fiscales et d'emploi.

Cette analyse s'est centrée sur la concentration des aides directes car le message 
politique de la CE sur la question de l'équité dans la réforme de 2014 était axé sur la répartition des paiements directs. Une analyse plus complète pourrait cependant être menée en l'élargissant aux mesures du second pilier de la PAC. Le principe d'équité constitue l'un des fondements centraux de la politique de développement

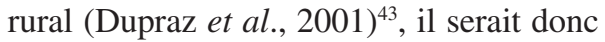
intéressant de prendre en compte certaines mesures comme l'indemnité compensatoire aux handicaps naturels ou encore de réfléchir dans le cas français sur la justification de maintenir un cadre national pour certaines mesures du développement rural visant à assurer plus d'équité dans le traitement des bénéficiaires entre régions.

Enfin, une analyse sur la contribution de l'inégalité des aides dans l'inégalité des revenus par actif agricole pourrait être menée pour approfondir ce travail. Les aides directes jouent un rôle déterminant dans la formation du revenu de nombreux agriculteurs qui en sont donc dépendants depuis longtemps (Blogowski et Chatellier, 2004) ${ }^{44}$. Un rapide aperçu à partir des données de 2011 des EM de l'UE, montre que la part des paiements directs dans le revenu est plus grande pour les exploitations agricoles dont la taille économique, approximée par la Production brute standard (PBS), est la plus importante. En moyenne, dans les EM où l'on trouve des EA dont la PBS est supérieure à $500000 €$, les aides directes représentent plus de $87 \%$ du revenu agricole net contre $45,3 \%$ pour les EA dont la taille économique est comprise entre $2000 €$ et $8000 €$ (calculs des auteures sur les données du Réseau d'information comptable agricole européen [RICA], 2011). Au-delà d'une convergence interne et externe des aides agricoles, une réflexion devra être menée sur la corrélation entre montant des aides versées, niveau et variabilité du revenu agricole hors aide, et nombre de travailleurs sur l'exploitation. Si la vocation des aides directes est effectivement le soutien du revenu, il est important que ces analyses soient disponibles. C'est sur cette base qu'une vraie réflexion pourra avoir lieu sur des règles d'attribution des aides qui contribuent plus efficacement à réduire les inégalités de revenu.
43. Ibid.

44. Blogowski A., Chatellier V. (2004). Les aides directes aux exploitations agricoles européennes et françaises depuis la réforme de la PAC de 1992. In J.-P. Butault (dir.). Les soutiens publics à l'agriculture : histoire, théorie, mesure. Paris, INRA Éditions, pp. 223-275.

\section{ANNEXES}

\section{Annexe 1. Code des pays}

BE Belgique ; BG Bulgarie ; $\mathrm{CZ}$ République tchèque, DK Danemark ; DE Allemagne ; EE Estonie ; EL Grèce ; ES Espagne ; FR France ; IE Irlande ; IT Italie ; CY Chypre ; LV Lettonie ; LT Lituanie ; LU Luxembourg ; HU Hongrie ; MT Malte ; NL Pays-Bas ; AT Autriche ; PL Pologne ; PT Portugal ; RO Roumanie ; SI Slovénie ; SK Slovaquie ; FI Finlande ; SE Suède ; UK Royaume-Uni. 


\begin{tabular}{|c|c|c|c|c|c|c|c|c|c|c|c|c|c|c|c|c|}
\hline 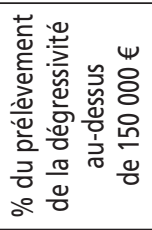 & & 0 & 0 & L & 요 & * & ○ & 음 & 응 & \begin{tabular}{|l}
$*$ \\
$*$ \\
L
\end{tabular} & 0 & L & in & in & 음 & n \\
\hline 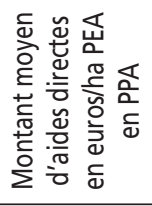 & $\stackrel{\mathscr{\Omega}}{\sim}$ & $\ddot{~}$ & ন্ & $\lesssim$ & 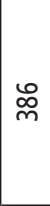 & 음 & 䎡 & $\bar{\infty}$ & $\stackrel{\text { I }}{\sim}$ & $\frac{d}{8}$ & $\stackrel{\stackrel{\sim}{\sim}}{\sim}$ & : & 导 & $\frac{d}{8}$ & $\tilde{\approx}$ & $\stackrel{\infty}{\circ}$ \\
\hline 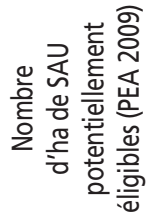 & $\begin{array}{l}\text { g } \\
\text { m } \\
6 \\
\overline{6}\end{array}$ & 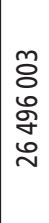 & 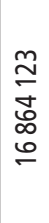 & 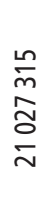 & $\begin{array}{l}\text { g } \\
\text { S } \\
\text { 음 } \\
0\end{array}$ & 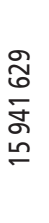 & 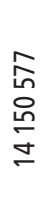 & $\begin{array}{l}\frac{m}{6} \\
m \\
0 \\
L \\
\omega\end{array}$ & $\begin{array}{l}\stackrel{0}{m} \\
\tilde{n} \\
\tilde{n} \\
\sigma\end{array}$ & $\begin{array}{l}\text { E } \\
\text { in } \\
6 \\
\text { Ln } \\
\text { in }\end{array}$ & 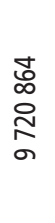 & 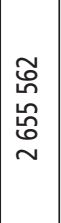 & $\begin{array}{l}\text { 옹 } \\
\frac{\operatorname{in}}{m} \\
m\end{array}$ & $\begin{array}{l}\text { Oे } \\
\text { 음 } \\
\infty \\
-\end{array}$ & 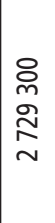 & 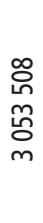 \\
\hline 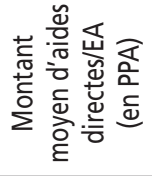 & 兴 & 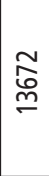 & $\underset{\substack{\infty \\
ٍ}}{0}$ & 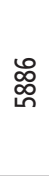 & $\underset{\widetilde{J}}{\stackrel{\Xi}{d}}$ & 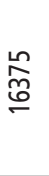 & ণ্ণি & $\stackrel{\text { 음 }}{\text { m }}$ & 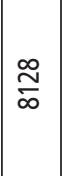 & 융 & ભ̊ & $\mid \begin{array}{l}0 \\
\hat{\tilde{\theta}} \\
0\end{array}$ & $\frac{\text { d }}{\text { in }}$ & $\begin{array}{l}\stackrel{L}{\infty} \\
\stackrel{0}{O}\end{array}$ & $\stackrel{\check{\gamma}}{\grave{\gamma}}$ & ঙั \\
\hline 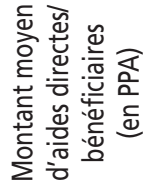 & ర్రి & 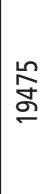 & 萑 & $\overline{0}$ & 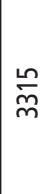 & ફ & 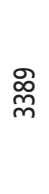 & $\underset{m}{\stackrel{\nabla}{m}}$ & $\frac{8}{\sigma}$ & $\stackrel{\stackrel{一}{m}}{=}$ & 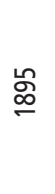 & 웅 & 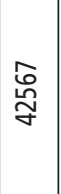 & 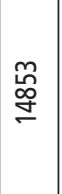 & 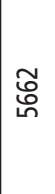 & $\underset{\infty}{\widetilde{\infty}}$ \\
\hline 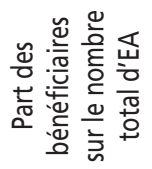 & 응 & $\tilde{\Omega}$ & $\begin{array}{l}m \\
o^{\circ} \\
0\end{array}$ & ळี & $\stackrel{\dot{m}}{m}$ & 芯 & ৪ั & సু & $\begin{array}{l}9 \\
\infty \\
\infty\end{array}$ & $\tilde{r}$ & $\stackrel{\text { Ln }}{\text { D }^{\circ}}$ & $\begin{array}{l}\text { ㅇ. } \\
\text { oo } \\
0\end{array}$ & : & శ్- & $\stackrel{\text { Ln }}{\stackrel{N}{N}}$ & ৪ి \\
\hline 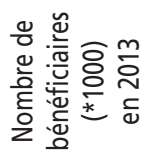 & $\begin{array}{l}\infty \\
\text { Oे } \\
\text { 守 }\end{array}$ & \begin{tabular}{|l}
$\bar{m}$ \\
$\mathbb{\sigma}$ \\
$\tilde{m}$
\end{tabular} & 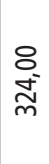 & $\begin{array}{l}\bar{\sigma} \\
\infty \\
\infty \\
\infty\end{array}$ & $\begin{array}{l}\frac{\sigma}{\sigma} \\
\stackrel{\infty}{=}\end{array}$ & $\frac{m}{\stackrel{m}{\infty}}$ & 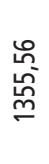 & 壱 & 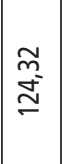 & $\underset{⿱}{\stackrel{5}{二}}$ & $\begin{array}{l}0 \\
0 \\
o \\
o \\
0\end{array}$ & $\begin{array}{c}\frac{P}{5} \\
\text { vo }\end{array}$ & $\stackrel{0}{0}$ & $\begin{array}{l}\infty \\
\stackrel{\infty}{n} \\
\stackrel{\circ}{n}\end{array}$ & $\stackrel{\stackrel{\Xi}{\infty}}{\stackrel{\Xi}{\Xi}}$ & $\begin{array}{l}\bar{\delta} \\
\text { Jे }\end{array}$ \\
\hline 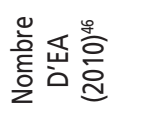 & 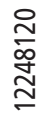 & $\frac{8}{6}$ & $\frac{\text { ㅇ }}{\text { হু }}$ & $\begin{array}{l}\text { ○ } \\
\text { ळ } \\
\text { ळ }\end{array}$ & 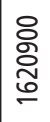 & 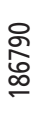 & 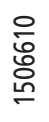 & $\begin{array}{l}\stackrel{0}{0} \\
\stackrel{D}{N}\end{array}$ & 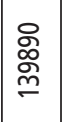 & $\begin{array}{l}0 \\
\infty \\
0 \\
\text { மn }\end{array}$ & 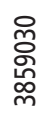 & $\frac{}{\check{z}}$ & $\begin{array}{c}\stackrel{0}{\infty} \\
\infty \\
\mathbb{N}\end{array}$ & 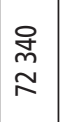 & $\stackrel{\infty}{\stackrel{\infty}{\circ}}$ & $\frac{8}{\frac{8}{1}}$ \\
\hline \multirow[t]{2}{*}{ 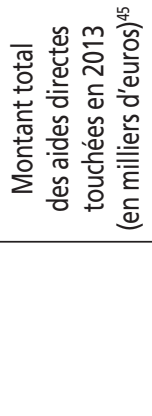 } & 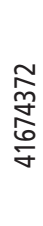 & $\begin{array}{l}\widetilde{0} \\
0 \\
0 \\
0 \\
\stackrel{0}{0} \\
\end{array}$ & 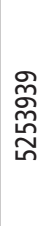 & 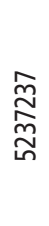 & $\begin{array}{l}\text { 음 } \\
\text { \%ু } \\
\text { ᄋ }\end{array}$ & 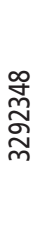 & 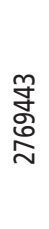 & $\begin{array}{l}\mathscr{Z} \\
\underset{\mathbb{D}}{\mathbb{N}} \\
\underset{\sim}{*}\end{array}$ & $\begin{array}{l}\stackrel{\Omega}{0} \\
\text { O̊ } \\
\stackrel{0}{\simeq}\end{array}$ & 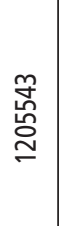 & $\begin{array}{l}m \\
\stackrel{m}{o} \\
o \\
o\end{array}$ & 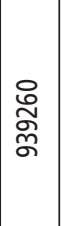 & $\underset{\infty}{\stackrel{\Xi}{\check{\Xi}}}$ & 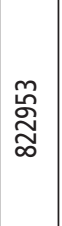 & 导 & 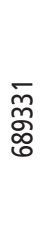 \\
\hline & ב્ב & 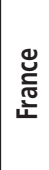 & 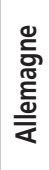 & 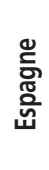 & 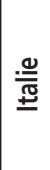 & 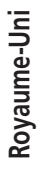 & $\begin{array}{l}\text { 동 } \\
\text { 응 }\end{array}$ & 惫 & 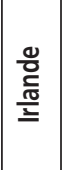 & 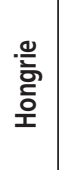 & 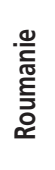 & 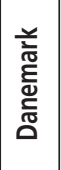 & 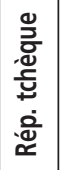 & 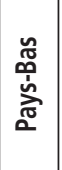 & 荡 & 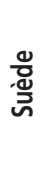 \\
\hline
\end{tabular}




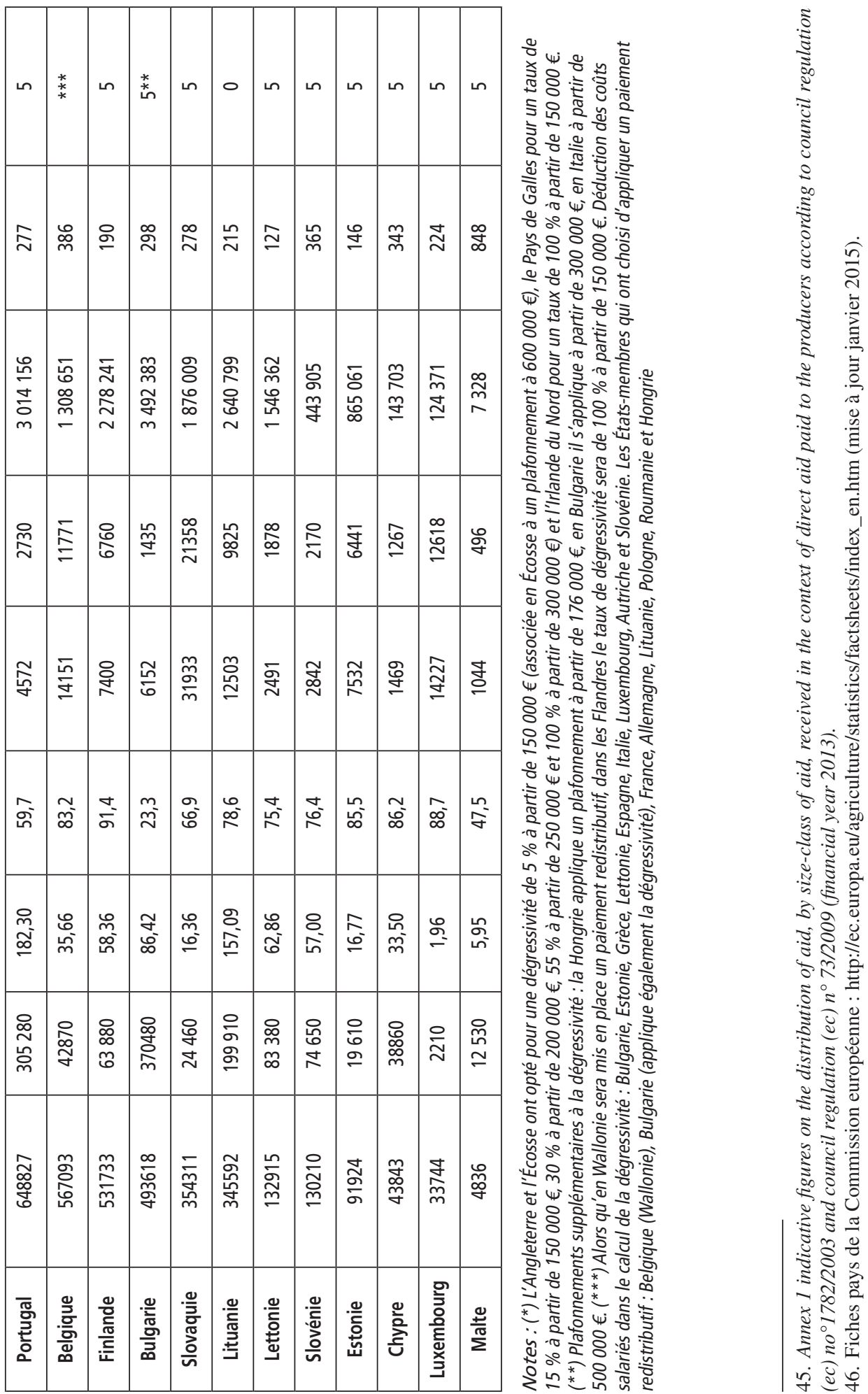

